\title{
In Vivo Tracing of Neural Tracts in the Intact and Injured Spinal Cord of Marmosets by Diffusion Tensor Tractography
}

\author{
Kanehiro Fujiyoshi, ${ }^{1,2 \star}$ Masayuki Yamada, ${ }^{4,5 \star}$ Masaya Nakamura, ${ }^{1}$ Junichi Yamane, ${ }^{1,2}$ Hiroyuki Katoh, ${ }^{1}$ \\ Kazuya Kitamura, ${ }^{1,2}$ Kenji Kawai, ${ }^{4}$ Seiji Okada, ${ }^{6}$ Suketaka Momoshima, ${ }^{3}$ Yoshiaki Toyama, ${ }^{1}$ and Hideyuki Okano ${ }^{2}$ \\ Department of ${ }^{1}$ Orthopaedic Surgery, ${ }^{2}$ Physiology, and ${ }^{3}$ Radiology and ${ }^{4}$ Center for Integrated of Medical Research, Keio University School of Medicine, \\ Shinjuku, Tokyo 160-8582, Japan, ${ }^{5}$ Central Institute for Experimental Animals, Miyamae-ku, Kawasaki, Kanagawa 216-0001, Japan, and ${ }^{6}$ Department of \\ Research Superstar Program Stem Cell Unit, Graduate School of Medical Science, Kyushu University, Fukuoka 812-8582, Japan
}

In spinal cord injury, axonal disruption results in motor and sensory function impairment. The evaluation of axonal fibers is essential to assess the severity of injury and efficacy of any treatment protocol, but conventional methods such as tracer injection in brain parenchyma are highly invasive and require histological evaluation, precluding clinical applications. Previous advances in magnetic resonance imaging technology have led to the development of diffusion tensor tractography (DTT) as a potential modality to perform in vivo tracing of axonal fibers. The properties and clinical applications of DTT in the brain have been reported, but technical difficulties have limited DTT studies of the spinal cord. In this study, we report the effective use of DTT to visualize both intact and surgically disrupted spinal long tracts in adult common marmosets. To verify the feasibility of spinal cord DTT, we first performed DTT of postmortem marmosets. DTT clearly illustrated spinal projections such as the corticospinal tract and afferent fibers in control animals, and depicted the severed long tracts in the injured animals. Histology of the spinal cords in both control and injured groups were consistent with DTT findings, verifying the accuracy of DTT. We also conducted DTT in live marmosets and demonstrated that DTT can be performed in live animals to reveal in vivo nerve fiber tracing images, providing an essential tool to evaluate axonal conditions in the injured spinal cord. Taken together, these findings demonstrate the feasibility of applying DTT to preclinical and clinical studies of spinal cord injury.

Key words: spinal cord injury; corticospinal tract; diffusion tensor tractography; magnetic resonance imaging; common marmoset; calmodulin-dependent protein kinase II- $\alpha$; pathway-specific DTT; in vivo tracing

\section{Introduction}

We established previously a reproducible spinal cord injury (SCI) model in adult common marmosets and demonstrated that transplantation of human neural stem/progenitor cells into the injured spinal cord promoted functional recovery (Iwanami et al., 2005b). An increase of axonal fibers, evaluated through histological methods, was observed near the transplanted neural stem/progenitor cells and were interpreted to be involved in the functional improvement. Such evaluation of axonal fibers is essential to assess the severity of SCI and efficacy of any treatment protocol (Olson, 2002; Kaneko et al., 2007), but conventional methods such as tracer injection in brain parenchyma are technically demanding and highly invasive (Ralston and Ralston, 1985; Lacroix et al., 2004). Because histological examinations are

\footnotetext{
Received July 24, 2007; accepted Sept. 4, 2007.

This work was supported by grants from the Leading Project for Realization of Regenerative Medicine from the Ministry of Education, Culture, Sports, Science and Technology (MEXT) of Japan, from the Japan Science and Technology Corporation, from the General Insurance Association, and by a grant-in-aid from the 21st Century COE Program of MEXT, Japan, to Keio University. We thank Hirotaka James Okano and Ichio Aoki for critical review of this manuscript, Humika Toyoda for tender animal care, and Ai Yokokawa for technical assistance.

*K.F. and M.Y. contributed equally to this work.

The authors declare no competing financial interests.

Correspondence should be addressed to Hideyuki Okano, 35 Shinanomachi, Shinjuku-ku, Tokyo 160-8582, Japan.E-mail: hidokano@sc.itc.keio.ac.jp.

DOI:10.1523/JNEUROSCI.3354-07.2007

Copyright $\odot 2007$ Society for Neuroscience $\quad 0270-6474 / 07 / 2711991-08 \$ 15.00 / 0$
}

required to evaluate tracer studies, it has been impossible to evaluate axonal fibers in vivo and follow the sequential growth of axonal fibers in the same animal. Understanding the value of such an examination method, we therefore sought to establish a noninvasive method to evaluate axonal fibers in vivo.

Magnetic resonance imaging (MRI) is essential for predicting prognosis and planning the treatment of patients with SCI (Kulkarni et al., 1987; Yamashita et al., 1990). Our previous study using common marmosets also demonstrated that MRI could detect pathological changes after SCI (Iwanami et al., 2005a). However, the information provided by conventional T1- and T2-weighted MRI of the spinal cord is essentially limited to the differentiation of the white matter from the gray matter. Conventional MRI depicts the white matter as a uniform tissue, although it actually contains a complex array of directionally oriented nerve fibers. Methods to visualize the pathways of the white matter in vivo have been long sought and, recently, diffusion tensor tractography (DTT) has demonstrated this ability (Ito et al., 2002; Masutani et al., 2003; Mori et al., 2003).

Diffusion tensor imaging (DTI) is a new imaging technique that takes advantage of the anisotropic nature of water diffusion in biological tissue to obtain detailed microstructural information (Le Bihan et al., 1986; Moseley et al., 1990; Basser et al., 1994; Beaulieu, 2002; Mori and Zhang, 2006). By analyzing and reconstructing that data obtained by DTI, DTT can follow the orienta- 

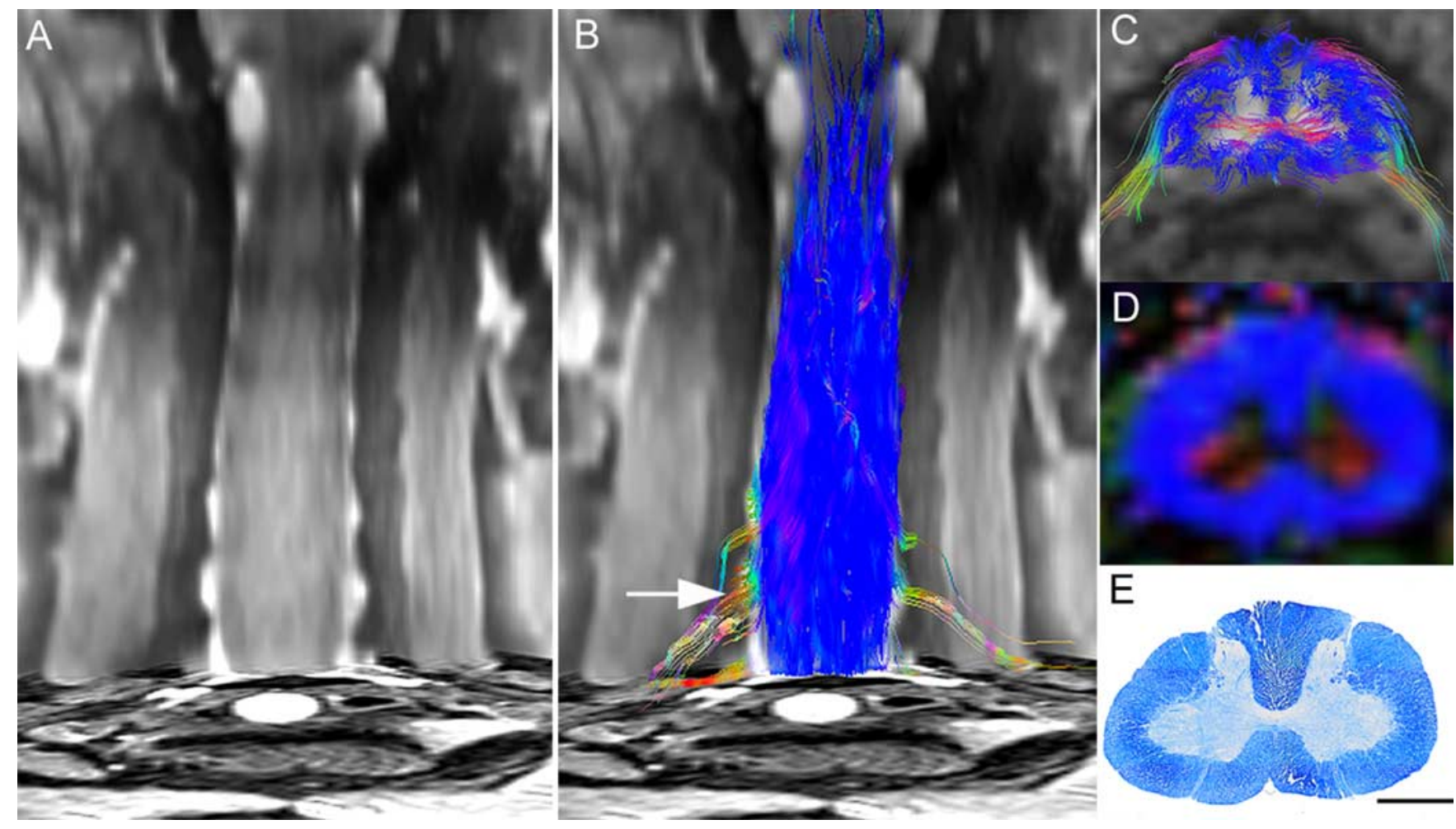

Figure 1. DTT of the intact spinal cord in a postmortem common marmoset. $\boldsymbol{A}$, Coronal T2-weighted MRI. $\boldsymbol{B}, \boldsymbol{C}$, Full-width DTT of the spinal cord ( $\boldsymbol{B}$ ) and an axial section $(\boldsymbol{C})$ superimposed on axial T2-weighted images. The ROI was placed in the lower cervical spinal cord and DTT was traced in the cranial direction. DTT tracts are color coded to indicate tract orientation: red for left-right orientation, green for anterior-posterior orientation, and blue for superior-inferior orientation. $\boldsymbol{D}, \boldsymbol{E}$, Directionally color-coded axial FA map of the spinal cord (D) reveals that the configuration of the white matter depicted in blue is consistent with the myelin-positive area in an axial section of the same area stained with LFB (E). $\boldsymbol{C}-\boldsymbol{E}$ are axial images of the $55 / 6$ level (B, arrow). Scale bar, 1 $\mathrm{mm}$.

tion of nerve fibers to trace specific neural pathways such as the corticospinal tract (CST) in the brain (Conturo et al., 1999; Masutani et al., 2003; Kamada et al., 2005a; Lee et al., 2005). Compared with the brain, however, DTT of the spinal cord is more difficult because of its smaller size and in vivo bulk motion (Basser and Jones, 2002; Maier and Mamata, 2005; Kharbanda et al., 2006). Several researchers have reported previously on successful DTT of the human spinal cord (Holder et al., 2000; Facon et al., 2005; Tsuchiya et al., 2005; Ducreux et al., 2006). However, because these DTT images were not confirmed with detailed histological studies, whether DTT actually reflects the anatomical axonal fibers remains unclear. In this study, we performed DTT of both intact and injured spinal cords in common marmosets and confirmed the accuracy of DTT through histology.

\section{Materials and Methods}

Hemisection SCI in common marmoset. Adult female common marmosets (266-384 g; Clea Japan, Tokyo, Japan) were used in the present study $(n=6)$. All interventions and animal care procedures were performed in accordance with the Laboratory Animal Welfare Act, the Guide for the Care and Use of Laboratory Animals (National Institutes of Health), and the Guidelines and Policies for Animal Surgery provided by the Animal Study Committee of the Central Institute for Experimental Animals of Keio University, and were approved by the ethics committee of Keio University. All surgeries were performed under general anesthesia induced by intramuscular injection of ketamine ( $50 \mathrm{mg} / \mathrm{kg}$; Sankyo, Tokyo, Japan) and xylazine ( $5 \mathrm{mg} / \mathrm{kg}$; Bayer, Leverkusen, Germany) and maintained by isoflurane (Foren; Abbott, Tokyo, Japan). The animal's pulse, arterial oxygen saturation, and rectal temperature were monitored during the surgical procedures. After a laminectomy at the C6 level, the dura mater was opened longitudinally and the right side of the spinal cord was cut at the C6 level using a surgical scalpel in the hemisection group $(n=$
3). The control group in this study in was a naive control without any surgical intervention.

Magnetic resonance imaging. MRI was performed using a 7.0 tesla MRI, PharmaScan 70/16 (BioSpin; Bruker) with a coil dedicated for small animals. In the studies using postmortem animals (control and hemisection groups, $n=2$ each), conventional T2-weighted images (T2WIs) were first obtained, followed by intracardiac perfusion with $4 \%$ paraformaldehyde (PFA), pH 7.4, and diffusion tensor MRI. T2WI and diffusion tensor MRI of the hemisected animals were conducted 2 weeks after injury. DTI data sets were acquired with a spin-echo sequence based on the Stejskal-Tanner diffusion preparation. Scanning parameters were as follows: repetition time (TR), $15000 \mathrm{~ms}$; echo time (TE), $40 \mathrm{~ms}$; flip angle, $90^{\circ}$, field of view (FOV), $55 \times 55 \mathrm{~mm}$; acquisition data matrix, $256 \times 256$; reconstructed image resolution, $0.215 \mathrm{~mm}$ (with zero-filling interpolation); slice thickness, $0.85 \mathrm{~mm}$; b-value, $1000 \mathrm{~s} / \mathrm{mm}^{2}$; motionprobing gradient (MPG) orientations, 12 axes; number of averaging (NA), 1. In the studies using live animals (control and hemisection group, $n=1$ each), conventional and diffusion tensor MRI were performed under the general anesthesia as mentioned above. MRI scans of the hemisected animal were conducted 2 weeks after injury. In live animals, to reduce motion artifacts from the blood flow and CSF flow, animals were immobilized on an acrylic bed with a specially designed head positioner and electrocardiogram (ECG) probe (SA Instruments) for gated imaging was attached to the animal's front thorax. DTI data sets in live animals were acquired with an ECG-gated standard diffusion weighted spin-echo pulse sequence based on the Stejskal-Tanner diffusion preparation (Stejskal and Tanner, 1965). Scanning parameters were as follows: TR, $3500 \mathrm{~ms}$; TE, $40 \mathrm{~ms}$; flip angle, $90^{\circ}$; FOV, $40 \times 40 \mathrm{~mm}$; acquisition data matrix, $128 \times 128$; reconstructed image resolution, $0.31 \times 0.31 \mathrm{~mm}$; slice thickness, $0.94 \mathrm{~mm}$; b-value, $1000 \mathrm{~s} / \mathrm{mm}^{2}$; MPG orientations, 12 axes; NA, 1.

Diffusion tensor analysis. Diffusion tensor and three-dimensional analysis were performed using Volume One and dTVIISR software (Kuni- 


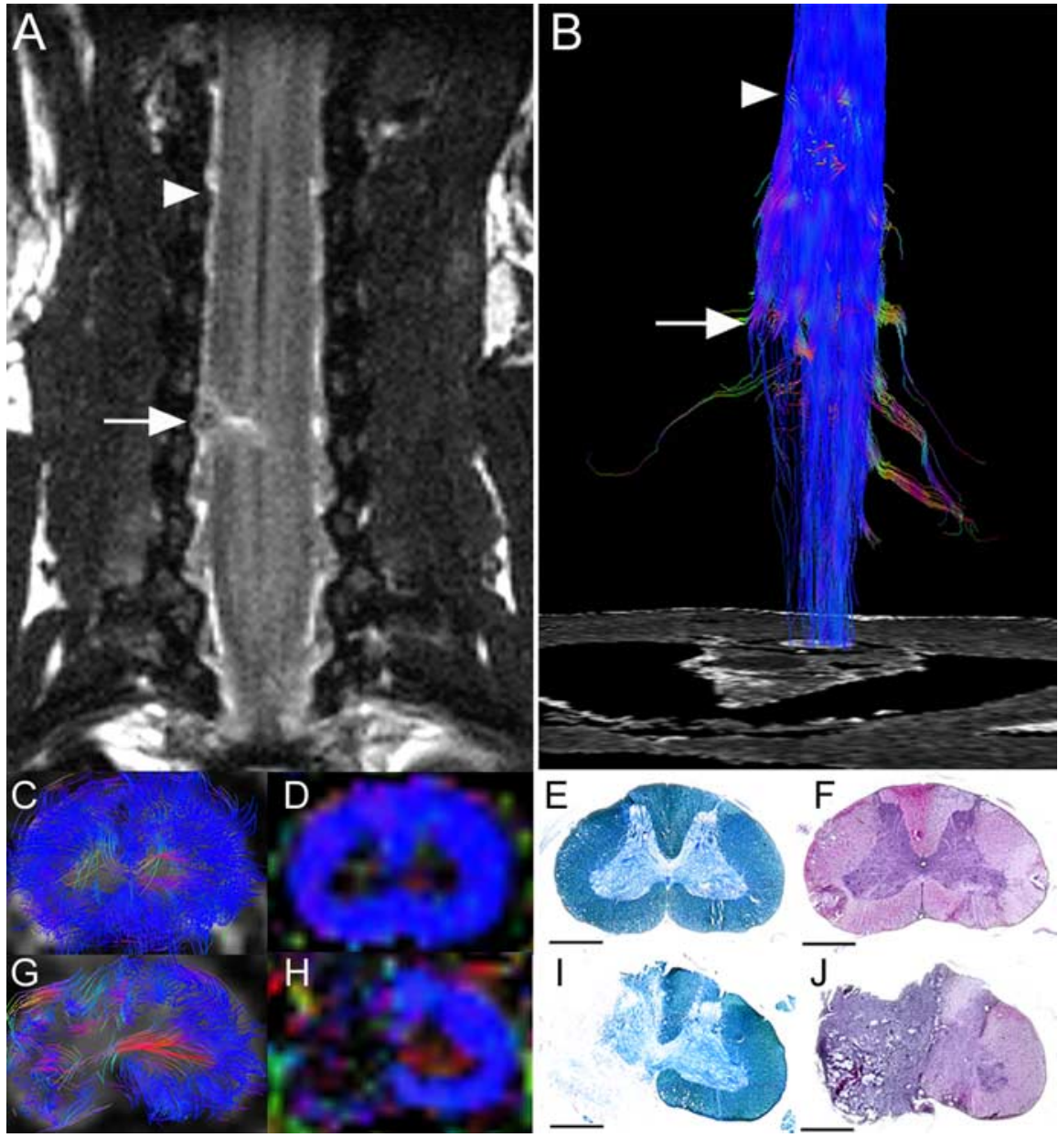

Figure 2. DTT of the hemisected spinal cord at 2 weeks after injury in a postmortem common marmoset. $A$, Coronal T2weighted MRI depicted the hemisection injury as a low-intensity area with no change in the cord caudal to the injury. $\boldsymbol{B}$, DTT of the hemisected spinal cord. The ROI was placed in the upper cervical spinal cord, and DTT was traced in the caudal direction revealing disruption of white matter fibers on the hemisected side. The traced tracts became untraceable at the injury site, whereas tracts on the contralateral side continued caudally. Arrows indicated the hemisection site and arrowheads indicated the point $8 \mathrm{~mm}$ cranial to the injury site in $\boldsymbol{A}$ and $\boldsymbol{B}$. $\boldsymbol{C}-\boldsymbol{J}, \mathrm{DTT}(\boldsymbol{C}, \boldsymbol{G})$, FA map $(\boldsymbol{D}, \boldsymbol{H})$, LFB staining $(\boldsymbol{E}, \boldsymbol{I})$, and HE staining $(\boldsymbol{F}, \boldsymbol{J})$ of the spinal cord $8 \mathrm{~mm}$ cranial to the injury site $(\boldsymbol{C} \boldsymbol{F})$ and at the hemisection site $(\mathbf{G} \boldsymbol{J})$. Although normal FA and anatomy of the spinal cord was confirmed cranial to the hemisection site, there was a significant decrease in FA of the white matter fibers at the hemisection site $(\mathbf{G}, \boldsymbol{H})$. Consistent with these changes in DTT $(\boldsymbol{G})$ and color-coded FA map $(\boldsymbol{H})$, demyelination was seen at the hemisection site $(\boldsymbol{I})$. Scale bars, $1 \mathrm{~mm}$.

matsu et al., 2003; Masutani et al., 2003). An eigenvector ( $e 1)$ associated with the largest eigenvalue $(\lambda 1)$ was assumed to represent the local fiber direction. Fiber tracking was initiated from a manually selected region of interest (ROI) area, which is the "seed" from which tracking lines were propagated bidirectionally according to the eigenvector $(e 1)$ at each voxel pixel. The direction of diffusion anisotropy was followed until tracking was terminated when it reached a voxel with a fractional anisotropy of $<0.25$. To delineate the motor tracts, the seed was placed on the area histologically known to contain CaMKII- $\alpha$-positive fibers in the upper cervical cord, which corresponds to the CST (Terashima et al., 1994; Iwanami et al., 2005a). To delineate the afferent pathways of the spinal cord, we placed the seed at the anterolateral and posterior funiculi of the lower cervical cord, which correspond to the spinothalamic tract and the gracile fasciculus, respectively. We used the two-regions-of-interest method (Mori et al., 2003), which consists of seed and target regions to depict the pyramidal decussation from the medullary pyramid (seed) to the opposite CST area in the upper cervical cord (target).

Histological analyses. In the postmortem group, spinal cord tissues were removed after diffusion tensor MRI. In the live group, each animal was perfused intracardially with $4 \%$ paraformaldehyde after diffusion tensor MRI and then the spinal cord tissues were removed. All spinal cord specimens were postfixed in $4 \%$ PFA and immersed overnight in 10\% sucrose followed by $30 \%$ sucrose. Frozen section blocks were prepared and cut into $20-\mu \mathrm{m}$-thick axial sections using a cryostat. These sections were stained with hematoxylin-eosin (HE) for general histological examinations and Luxol fast blue (LFB) for evaluation of the myelinated area. Immunostaining with anti-CaMKII- $\alpha$ antibody (primary antibody, diluted 1:100, mouse monoclonal; Zymed, San Francisco, CA; secondary antibody, a biotin-labeled goat anti-mouse IgG for ABC and DAB staining) was performed to examine the CST.

\section{Results \\ DTT of intact and injured spinal cords in postmortem common marmosets} Based on the data from in vivo highresolution diffusion tensor MR images of postmortem common marmoset spinal cords (Fig. $1 A$ ), we created a DTT of the cervical spinal cord that enabled us to detect various fibers of the spinal cord (Fig. $1 B)$. In a color-coded DTT of the intact cervical spinal cord, each path traced by DTT, which we will refer to have as a tract, is depicted in colors according to its orientation (Pajevic and Pierpaoli, 1999): red for left-right orientation, green for anterior-posterior orientation, and blue for superior-inferior orientation (Fig. $1 B, C$ ). White matter fibers with high craniocaudal diffusion anisotropy were visible in blue on color-coded maps of fractional anisotropy (FA) (Basser and Pierpaoli, 1996), an index of anisotropy ranging from 0 (perfectly isotropic diffusion) to 1 (a hypothetical infinite cylinder), which had been calculated from the same data (Fig. 1D) and the distribution of longitudinal fibers was consistent with the distribution of myelinated axons stained with LFB (Fig. $1 E$ ). In the gray matter, horizontal fibers passing from the central canal to the anterior horn of the spinal cord were observed in red, but almost no diffusion anisotropy was detected in the remaining gray matter, which is mainly occupied by neuronal and glial cells.

In the marmosets with hemisected spinal cords, the hemisected area of spinal cord appeared as a high-intensity area in coronal T2WIs 2 weeks after injury (Fig. 2A). DTT of the hemisected spinal cord revealed that the longitudinal fibers of the white matter in the injured side were disrupted at the hemisection site, but preserved in the intact side (Fig. $2 B$ ). In axial DTT images and color-coded FA maps, there was a significant decrease in FA of the longitudinal fibers of the white matter as well as the transverse fibers of the gray matter at the hemisection site (Fig. $2 \mathrm{G}, H)$, compared with the site $8 \mathrm{~mm}$ cranial to the hemisection site (Fig. 2C,D). Consistent with these changes in DTT and colored FA maps, histology of the hemisected area revealed disruption of both gray and white matter with severe demyelination. (Fig. $2 I, J)$. 


\section{Pathway-specific DTT in postmortem} common marmosets

To examine the feasibility of visualizing individual pathways in common marmosets, we analyzed the CST from the medulla to the upper cervical spinal cord because the course of the CST in this region is unique and well known. Analysis of histological sections stained for calmodulindependent protein kinase II- $\alpha$ (CaMKII- $\alpha$ ) determined the location of the CST (Terashima et al., 1994; Iwanami et al., 2005a) within the medulla, and DTT of the CST was performed by setting the ROI, which is a manually selected area based on anatomical knowledge from which DTT fiber tracking was initiated, in the pyramid of the medulla and tracing caudally (Fig. 3A). The course of the CST in this area has been well documented in the literature; the majority of fibers cross to the contralateral side through the pyramidal decussation and descended the lateral funiculus (lateral CST), whereas a small group of fibers descend the ipsilateral anterior (anterior CST) and lateral funiculus (anterolateral CST) (Qiu et al., 1991; Lacroix et al., 2004; Lemon et al., 2004). A study in humans demonstrated the composition of the CST, with the lateral CST accounting for $90 \%$ of CST fibers and the anterior CST and uncrossed lateral CST accounting for the remaining 8 and $2 \%$, respectively (Carpenter and Sutin, 1983). DTT was capable of tracing fibers through all three courses and successfully demonstrated the pyramidal decussation of the CST (Fig. 3B-E). However the number of tracts depicted with DTT through each course did not reflect the amount of CST fibers traveling through each pathway, because the number of DTT tracts depicted descending each pathway were similar although a majority of CST fibers actually travel through the lateral CST.

We focused on the pyramidal decussation of the CST and conducted histological studies to verify the results of DTT. DTT and histological sections from similar points along the craniocaudal axis were compared. The course of the CST depicted by DTT (Fig. $3 H$ ) corresponded to the area positive for CaMKII- $\alpha$ (Fig. 3I), which recognizes fibers of the CST, and LFB staining of the same section (Fig. 3J) confirmed that the CST delineated by DTT and CaMKII- $\alpha$ contained myelinated fibers. CST-specific DTT superimposed on MR images verified that the pyramidal decussation was depicted in the proper position (Fig. 3F, G).

With sufficient data to indicate that CST-specific DTT is possible, we then con-
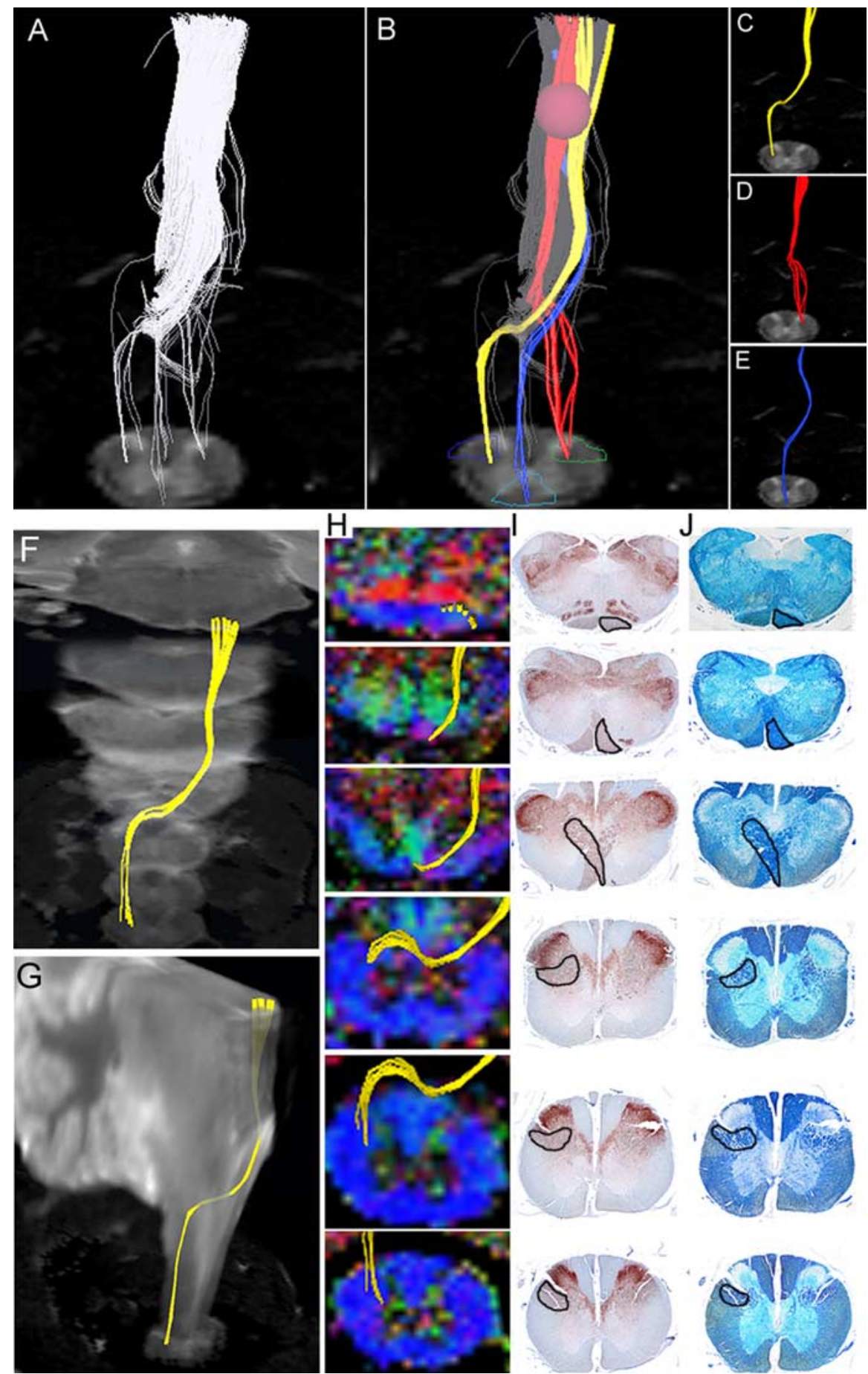

Figure 3. Pathway-specific DTT in a postmortem common marmoset revealing the course of the corticospinal tract with pyramidal decussation. $A$, DTT of the CST was conducted by placing the ROl in the pyramid of the medulla and tracing caudally. Note that the volume of traced tracts decrease as the tracing was carried caudally, because many tracts became untraceable because of the partial volume effect. $\boldsymbol{B}$, By placing secondary ROIs in areas of the upper cervical spinal cord known to contain CST fibers, CST fibers that pass through both ROls could be depicted. C, Lateral CST fibers that crossed over to and descended the contralateral lateral funiculus in a pattern suggesting pyramidal decussation were depicted in yellow. $\boldsymbol{D}$, Uncrossed lateral fibers descending the ipsilateral lateral funiculus were depicted in red. $\boldsymbol{E}$, Lateral fibers descending the ipsilateral anterior funiculus were depicted in blue. The fact that DTT was capable of accurately depicting all three known pathways of the CST is significant. However, it is important to note that the depicted DTT tracts do not accurately reflect the volume of nerve fibers, because it is known that the lateral CST contains the majority of CST fibers. F, G, DTT of the pyramidal decussation superimposed on three-dimensional MR images to macroscopically confirm that the pyramidal decussation was depicted in the proper height in the medulla and the upper cervical cord, using the cerebellum as a reference point. $\boldsymbol{H}, \mathrm{DTT}$ of the pyramidal decussation superimposed on axial color-coded FA maps. $\boldsymbol{I}, \boldsymbol{J}$, Axial histological slices of the same points in $\boldsymbol{H}$ stained for CaMKII- $\alpha$ to reveal the location of CST fibers $(\boldsymbol{I})$ and LFB to delineate the configuration of the white matter $(\boldsymbol{J})$. In each slice, the area through which the DTT CST tract passes was positive for CaMKII- $\alpha$ and LFB, confirming the accuracy of DTT. 

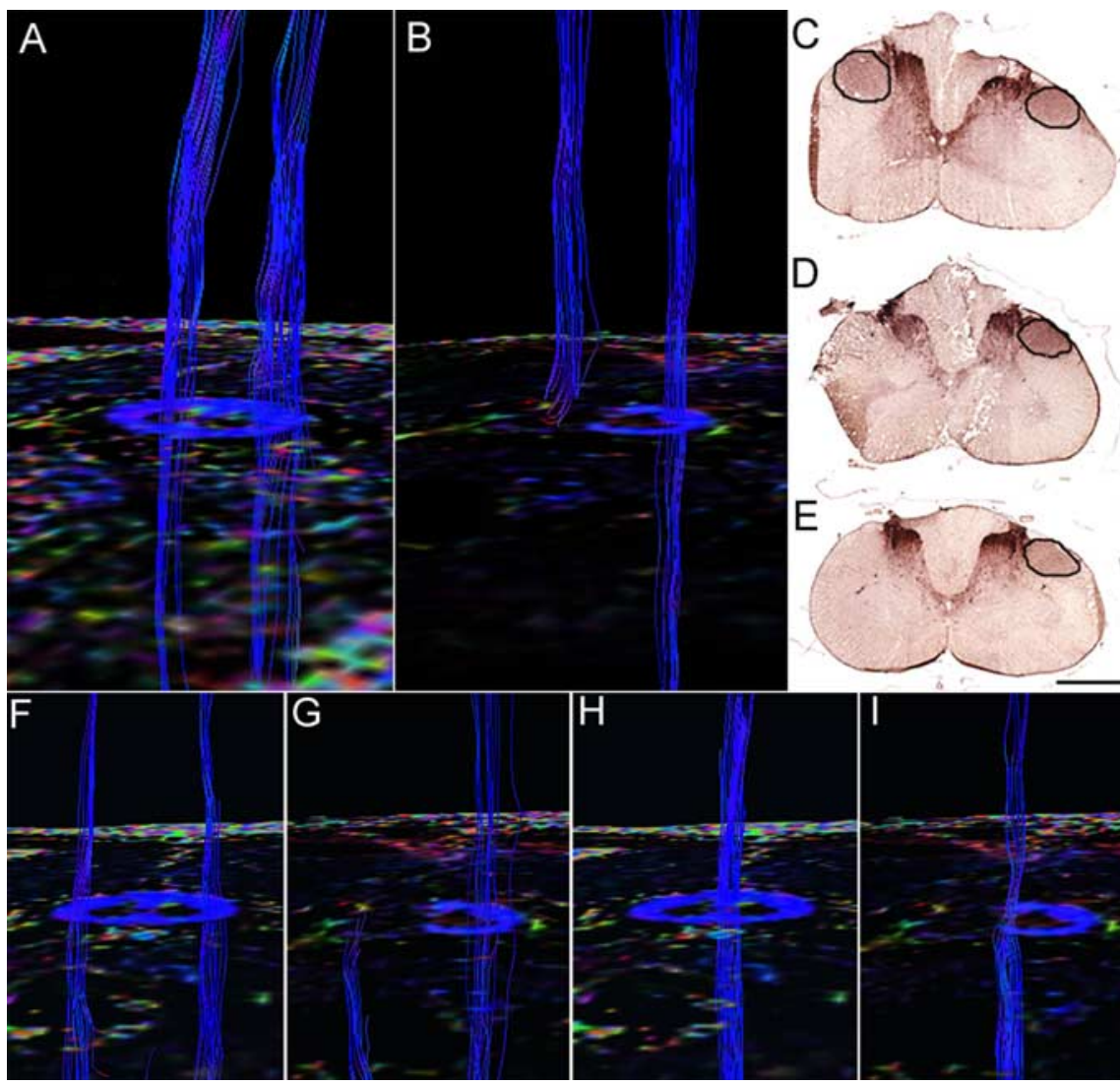

Figure 4. In vivo CST- and afferent pathway-specific DTT of intact and injured spinal cords in postmortem common marmosets. $\boldsymbol{A}$, DTT of an intact CST superimposed on a color-coded FA map of the C5/6 level. $\boldsymbol{B}$, DTT of the CST in a hemisected spinal cord superimposed on a color-coded FA map of the hemisected C5/6 level 2 weeks after injury revealing disruption of the CST at the site of injury. $\boldsymbol{C}-\boldsymbol{E}$, (aMKIl- $\alpha$ staining of an axial section of the hemisected spinal cord $8 \mathrm{~mm}$ cranial to the hemisection site $(\boldsymbol{C})$, at the hemisection site $(\boldsymbol{D})$, and $8 \mathrm{~mm}$ caudal to the hemisection site $(\boldsymbol{E})$. Because CaMKII- $\alpha$ is a known substance transported in the axons of the CST, the absence of CaMKII- $\alpha$ staining distal to the injury suggested CST disruption, confirming the results of CST-specific DTT. $\boldsymbol{F}, \boldsymbol{H}$, In the control group, ROI was placed at the anterolateral funiculus $(\boldsymbol{F})$ or the posterior funiculus $(\boldsymbol{H})$, and a DTT of the afferent pathways was drawn in the caudocranial direction. $\mathbf{G}, \boldsymbol{I}, \mathbf{I n}$ the hemisection group, no fibers were observed at the site rostral to the hemisection site, demonstrating that tract-specific DTT can potentially delineate the spinothalamic tract $(\boldsymbol{G})$ and dorsal column-medial lemniscus pathway (I). Scale bar, $1 \mathrm{~mm}$.

ducted CST-specific DTT of hemisected spinal cords to observe how DTT would depict an injured pathway. CST-specific DTTs of the middle to lower cervical spinal cord were compared in intact and hemisected postmortem common marmosets. In the intact cervical spinal cord, DTT depicted descending CST tracts in the bilateral lateral funiculus (Fig. $4 D$ ). In the hemisected cervical spinal cord 2 weeks after injury, FA decreased in colorcoded FA maps and no tracts were found caudal to the hemisection site on the injured side whereas the intact CST pathway was observed descending the uninjured side (Fig. 4B). Histology confirmed the disruption of the CST at the hemisection site with robust CaMKII- $\alpha$ staining rostral to the hemisection (Fig. $4 C$ ) and no CaMKII- $\alpha$ positive CST fibers at and caudal to the hemisection site (Fig. 4D,E).

Using a similar technique, it is also possible to trace pathways other than the CST. By placing the ROI in areas known to contain afferent fibers and tracing in the caudocranial direction, the spinothalamic tract in the anterolateral funiculus (Fig. $4 F$ ) and the medial lemniscus pathway in the posterior funiculus (Fig. 4B) were depicted. When the same procedure was repeated in the hemisected group, the ascending tracts were disrupted at the hemisection site with no tracts rostral to the lesion on the injured side, whereas normal ascending tracts were observed in the uninjured side (Fig. $4 G, I)$.

\section{Pathway-specific DTT of intact and injured spinal cords in live common marmosets}

To evaluate the clinical feasibility of DTT, we repeatedly performed in vivo pathwayspecific DTT on live animals and compared the results with those obtained from postmortem animals. Similar to the DTT of postmortem animals, in vivo DTT of the intact cervical CST in live animals showed longitudinal tracts in the lateral funiculus bilaterally (Fig. 5B), and pathway-specific DTT of afferent fibers depicted tracts in the anterolateral and posterior funiculus (Figs. 5C,D). In the hemisected marmoset 2 weeks after injury, T2WI MRI revealed a low-intensity area at the hemisection site and a high-intensity area at the same level on the intact side (Fig. $5 E$ ), whereas DTT showed disruption of the CST (Fig. 5F) and ascending fibers at the lesion site (Fig. $5 G, H)$. Pathway-specific in vivo DTT findings in live animals were highly similar to those of postmortem animals, especially in major tract morphology. Because MRI scans of live animals required anesthesia, the scan duration and, therefore, scan area were considerably limited compared with postmortem animals; MRIs of live animals were conducted in $1.5 \mathrm{~h}$ whereas $10 \mathrm{~h}$ scans were performed for postmortem animals. Overall, these findings demonstrated that it is feasible to depict the descending and ascending pathways of the spinal cord in live animals using pathway-specific DTT, and demonstrate the usefulness of DTT as an imaging method to assess specific path-

ways in spinal cord injuries.

\section{Discussion}

Because we hope to clinically apply this procedure to human SCI patients in the future, common marmosets were selected for this study. As primates, they are closely related to humans in terms of neurofunctional anatomy of the spinal cord. For example, the CST fibers localize mainly in the dorsal funiculus in rodents, whereas in primates they are mainly located in the lateral funiculus (Qiu et al., 1991; Terashima et al., 1994; Lacroix et al., 2004; Lemon et al., 2004; Iwanami et al., 2005a). From a practical standpoint, common marmosets are easy to handle, breed effectively, and are small enough to fit into the narrow MRI coil.

In our past studies of SCI in common marmosets, we have used contusion injury models because, compared with other SCI methods, contusion injuries more closely resemble the pathological conditions found in human SCI patients (Iwanami et al., 2005a,b). In this study, however, we chose a hemisection model because the disruption and regeneration of axons after a hemisection injury is easier to evaluate than a contusion injury (Levi et al., 2002; Tuszynski et al., 2002). Because the main objective of this study was to evaluate the usefulness of DTT in assessing axonal 


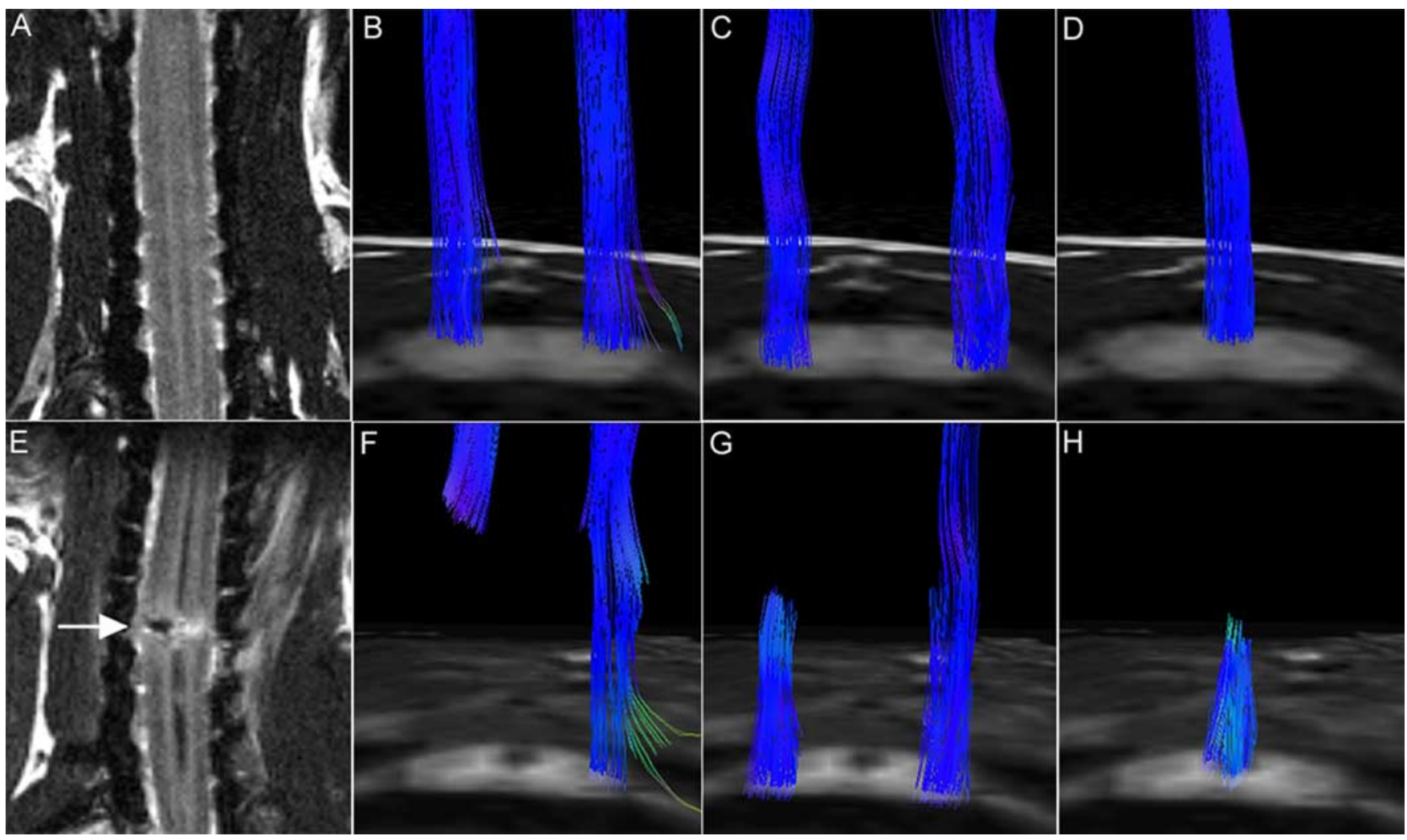

Figure 5. $\boldsymbol{A}-\boldsymbol{H}$, In vivo pathway-specific DTT of intact and injured spinal cords in live common marmosets. MRI and tract-specific DTT of the intact spinal cord $(\boldsymbol{A}-\boldsymbol{D})$ and hemisected spinal cord 2 weeks after injury $(\boldsymbol{E}-\boldsymbol{H})$. DTTs of the $C S T(\boldsymbol{B}, \boldsymbol{F})$, spinothalamic tract $(\boldsymbol{C}, \boldsymbol{G})$, and dorsal column-medial lemniscus pathway $(\boldsymbol{D}, \boldsymbol{H})$ were conducted in both groups, revealing tract disruption at the hemisection site (C5/6 level) in all pathways. Although there are some limitations, pathway-specific in vivo DTT conducted in live animals yielded results similar to those observed in postmortem animals, especially in respect to major tract morphology.

conditions in SCI and to confirm the accuracy of DTT by comparing DTT images with histological findings, an injury with less complexity and ambiguousness was desired. With the convincing images obtained in this study, it would be interesting to examine contusion injury models in the future.

With the ability to visualize axonal projections in threedimensions, DTT has tremendous potential as a tool to diagnose and evaluate CNS disease and trauma. In fact, DTT is already being clinically applied to visualize cerebral long tracts in cerebral surgery (Kamada et al., 2005b; Okada et al., 2006). Although there have been several preliminary studies of spinal cord DTI and DTT, they have not fully explored the potential of DTI technology. One reason DTI of the spinal cord has been less studied compared with the brain is the technical difficulty involved in conducting imaging of the spinal cord. DTI of the spinal cord requires high spatial resolution, is easily affected by magnetic susceptibility, and is obscured by in vivo bulk motion brought about by the beating of the heart, respiration, and the flow of CSF (Basser and Jones, 2002; Maier and Mamata, 2005; Kharbanda et al., 2006). In the present study, a 7.0 tesla MRI was used to obtain images with high resolution and a spin echo protocol was used to minimize magnetic susceptibility. To eliminate the effect of in vivo bulk motion, we first conducted our study using postmortem animals. Because a previous study demonstrated a degradation of diffusion anisotropy in the postmortem spinal cord (Matsuzawa et al., 1995; Madi et al., 2005), we performed all imaging immediately after animals were killed. By using postmortem animals it was possible to conduct scans of long duration (an average scan time of $10 \mathrm{~h}$ ), resulting in images with high spatial resolution.
In our study using live animals, all animals were maintained under general anesthesia and cardiac-gated imaging was incorporated to minimize the effects of bulk motion. Under general anesthesia, marmosets were immobilized on an acrylic bed with a specially designed head positioner. Because the total scan duration was limited by anesthetic considerations, scan time (average $1.5 \mathrm{~h}$ ) and, therefore, scan area and spatial resolution were limited compared with postmortem animal studies. However, it is of enormous importance that DTT of a live animal was able to visualize intact neural pathways and also the disrupted pathways in an injured animal, because this is the only method currently available or in development that can reveal in vivo axonal pathways.

In this study, we focused mainly on the CST to conduct pathway-specific DTT because it is the most important pathway in terms of motor function and often becomes the subject of scrutiny in studies of spinal cord injury treatment protocols. CST-specific DTT accurately depicted the course of the CST from the medulla to the cervical spinal cord and succeeded in imaging the "pyramidal decussation," which has been considered difficult to visualize. Furthermore, CST-specific DTT of the hemisected animal revealed the disruption of the CST at the site of injury. By using the dTV DTT software (Kunimatsu et al., 2003; Masutani et al., 2003), it is also possible to set the ROI at any point of interest and to perform voxel unit fiber tracking from that position within the threshold limit set for diffusion anisotropy. This allowed us to conduct DTT of the afferent pathways in both intact and injured spinal cords, illustrating the enormous value of this method. This capability to visualize specific projections can be applied to various studies of the spinal cord. For example, an 
interesting study would be a study of ascending projections and its involvement in allodynia, using functional MRI to assess sensory dysfunction (Hofstetter et al., 2005; Lilja et al., 2006).

DTT is a new technique that traces white matter fiber trajectories by tracking the direction of faster diffusion, which is assumed to correspond to the longitudinal axis of the tract. However it is important to keep in mind that the tracking is conducted in units called voxels, which, in this study, is $0.215 \mathrm{~mm}$ in size, considerably larger than any one individual axonal tract. Therefore, what is actually being tracked is a group of axonal fibers with perhaps some tissue other than the intended fibers at times included in the same voxel (Mori and van Zijl, 2002; Mori and Zhang, 2006). When tissues other than the targeted axonal tract are present within the same voxel, their diffusion anisotropy interferes destructively in a phenomenon referred to as partial volume effect (Alexander et al., 2001). For example, if multiple axonal fiber tracts with different trajectories cross within the same voxel, their diffusion anisotropy becomes merged and may become more isotropic, losing directional information. The tracking procedure is often terminated because the path comes to a voxel that has lost directional orientation (anisotropy) as a result of this partial volume effect (Fig. 3A-E). Partial volume effect can also result in a misleading redirection of anisotropy, leading to incorrect fiber tracking. It is also important to understand that the number of tracts traced by DTT does not necessarily reflect the actual volume of white matter fiber trajectories (Fig. $3 A-E$ ).

With the convincing images obtained in this study, the possibilities and the limitations of spinal cord DTT need to be further explored. For example, the next step would be DTT of contusion SCI models. Another significant point that needs to be studied, is whether DTT has the sensitivity to detect regenerating axons. If confirmed, DTT would allow tracing studies at multiple time points in the same animal/patient, becoming an indispensable tool to monitor and evaluate the effectiveness of any treatment protocol for spinal cord injury. Whatever the results reveal, DTT of the spinal cord is a powerful tool with tremendous potential if its properties and limitations are fully understood and correctly applied.

\section{References}

Alexander AL, Hasan KM, Lazar M, Tsuruda JS, Parker DL (2001) Analysis of partial volume effects in diffusion-tensor MRI. Magn Reson Med 45:770-780.

Basser PJ, Jones DK (2002) Diffusion-tensor MRI: theory, experimental design and data analysis - a technical review. NMR Biomed 15:456-467.

Basser PJ, Pierpaoli C (1996) Microstructural and physiological features of tissues elucidated by quantitative-diffusion-tensor MRI. J Magn Reson B 111:209-219.

Basser PJ, Mattiello J, LeBihan D (1994) MR diffusion tensor spectroscopy and imaging. Biophys J 66:259-267.

Beaulieu C (2002) The basis of anisotropic water diffusion in the nervous system-a technical review. NMR Biomed 15:435-455.

Carpenter MB, Sutin J (1983) Human neuroanatomy, Ed 8, pp 282-289. Baltimore: Lippincott, Williams and Wilkins.

Conturo TE, Lori NF, Cull TS, Akbudak E, Snyder AZ, Shimony JS, McKinstry RC, Burton H, Raichle ME (1999) Tracking neuronal fiber pathways in the living human brain. Proc Natl Acad Sci USA 96:10422-10427.

Ducreux D, Lepeintre JF, Fillard P, Loureiro C, Tadie M, Lasjaunias P (2006) MR diffusion tensor imaging and fiber tracking in 5 spinal cord astrocytomas. AJNR Am J Neuroradiol 27:214-216.

Facon D, Ozanne A, Fillard P, Lepeintre JF, Tournoux-Facon C, Ducreux D (2005) MR diffusion tensor imaging and fiber tracking in spinal cord compression. AJNR Am J Neuroradiol 26:1587-1594.

Hofstetter CP, Holmstrom NA, Lilja JA, Schweinhardt P, Hao J, Spenger C, Wiesenfeld-Hallin Z, Kurpad SN, Frisen J, Olson L (2005) Allodynia limits the usefulness of intraspinal neural stem cell grafts; directed differentiation improves outcome. Nat Neurosci 8:346-353.
Holder CA, Muthupillai R, Mukundan Jr S, Eastwood JD, Hudgins PA (2000) Diffusion-weighted MR imaging of the normal human spinal cord in vivo. AJNR Am J Neuroradiol 21:1799-1806.

Ito R, Mori S, Melhem ER (2002) Diffusion tensor brain imaging and tractography. Neuroimaging Clin N Am 12:1-19.

Iwanami A, Yamane J, Katoh H, Nakamura M, Momoshima S, Ishii H, Tanioka Y, Tamaoki N, Nomura T, Toyama Y, Okano H (2005a) Establishment of graded spinal cord injury model in a nonhuman primate: the common marmoset. J Neurosci Res 80:172-181.

Iwanami A, Kaneko S, Nakamura M, Kanemura Y, Mori H, Kobayashi S, Yamasaki M, Momoshima S, Ishii H, Ando K, Tanioka Y, Tamaoki N, Nomura T, Toyama Y, Okano H (2005b) Transplantation of human neural stem cells for spinal cord injury in primates. J Neurosci Res 80:182-190.

Kamada K, Todo T, Morita A, Masutani Y, Aoki S, Ino K, Kawai K, Kirino T (2005a) Functional monitoring for visual pathway using real-time visual evoked potentials and optic-radiation tractography. Neurosurgery 57 [Suppl]:121-127.

Kamada K, Todo T, Masutani Y, Aoki S, Ino K, Takano T, Kirino T, Kawahara N, Morita A (2005b) Combined use of tractography-integrated functional neuronavigation and direct fiber stimulation. J Neurosurg 102:664-672.

Kaneko S, Iwanami A, Nakamura M, Kishino A, Kikuchi K, Shibata S, Okano HJ, Ikegami T, Moriya A, Konishi O, Nakayama C, Kumagai K, Kimura T, Sato Y, Goshima Y, Taniguchi M, Ito M, He Z, Toyama Y, Okano H (2007) A selective Sema3A inhibitor enhances regenerative responses and functional recovery of the injured spinal cord. Nat Med 12:1380-1389.

Kharbanda HS, Alsop DC, Anderson AW, Filardo G, Hackney DB (2006) Effects of cord motion on diffusion imaging of the spinal cord. Magn Reson Med 56:334-339.

Kulkarni MV, Williams JC, Yeakley JW, Andrews JL, McArdle CB, Narayana PA, Howell RR, Jonas AJ (1987) Magnetic resonance imaging in the diagnosis of the cranio-cervical manifestations of the mucopolysaccharidoses. Magn Reson Imaging 5:317-323.

Kunimatsu A, Aoki S, Masutani Y, Abe O, Mori H, Ohtomo K (2003) Threedimensional white matter tractography by diffusion tensor imaging in ischaemic stroke involving the corticospinal tract. Neuroradiology 45:532-535.

Lacroix S, Havton LA, McKay H, Yang H, Brant A, Roberts J, Tuszynski MH (2004) Bilateral corticospinal projections arise from each motor cortex in the macaque monkey: a quantitative study. J Comp Neurol 473:147-161.

Le Bihan D, Breton E, Lallemand D, Grenier P, Cabanis E, Laval-Jeantet M (1986) MR imaging of intravoxel incoherent motions: application to diffusion and perfusion in neurologic disorders. Radiology 161:401-407.

Lee JS, Han MK, Kim SH, Kwon OK, Kim JH (2005) Fiber tracking by diffusion tensor imaging in corticospinal tract stroke: topographical correlation with clinical symptoms. NeuroImage 26:771-776.

Lemon RN, Kirkwood PA, Maier MA, Nakajima K, Nathan P (2004) Direct and indirect pathways for corticospinal control of upper limb motoneurons in the primate. Prog Brain Res 143:263-279.

Levi AD, Dancausse H, Li X, Duncan S, Horkey L, Oliviera M (2002) Peripheral nerve grafts promoting central nervous system regeneration after spinal cord injury in the primate. J Neurosurg 96:197-205.

Lilja J, Endo T, Hofstetter C, Westman E, Young J, Olson L, Spenger C (2006) Blood oxygenation level-dependent visualization of synaptic relay stations of sensory pathways along the neuroaxis in response to graded sensory stimulation of a limb. J Neurosci 26:6330-6336.

Madi S, Hasan KM, Narayana PA (2005) Diffusion tensor imaging of in vivo and excised rat spinal cord at $7 \mathrm{~T}$ with an icosahedral encoding scheme. Magn Reson Med 53:118-125.

Maier SE, Mamata H (2005) Diffusion tensor imaging of the spinal cord. Ann NY Acad Sci 1064:50-60.

Masutani Y, Aoki S, Abe O, Hayashi N, Otomo K (2003) MR diffusion tensor imaging: recent advance and new techniques for diffusion tensor visualization. Eur J Radiol 46:53-66.

Matsuzawa H, Kwee IL, Nakada T (1995) Magnetic resonance axonography of the rat spinal cord: postmortem effects. J Neurosurg 83:1023-1028.

Mori H, Masutani Y, Aoki S, Abe O, Hayashi N, Masumoto T, Yamada H, Yoshikawa T, Kunimatsu A, Ohtomo K, Kabasawa H (2003) [Simple visualization of the corticospinal pathway using tractography: one-ROI 
and two-ROI methods]. Nippon Igaku Hoshasen Gakkai Zasshi 63:51-53.

Mori S, van Zijl PC (2002) Fiber tracking: principles and strategies—a technical review. NMR Biomed 15:468-480.

Mori S, Zhang J (2006) Principles of diffusion tensor imaging and its applications to basic neuroscience research. Neuron 51:527-539.

Moseley ME, Cohen Y, Kucharczyk J, Mintorovitch J, Asgari HS, Wendland MF, Tsuruda J, Norman D (1990) Diffusion-weighted MR imaging of anisotropic water diffusion in cat central nervous system. Radiology 176:439-445

Okada T, Mikuni N, Miki Y, Kikuta K, Urayama S, Hanakawa T, Fushimi Y, Yamamoto A, Kanagaki M, Fukuyama H, Hashimoto N, Togashi K (2006) Corticospinal tract localization: integration of diffusion-tensor tractography at 3-T MR imaging with intraoperative white matter stimulation mapping-preliminary results. Radiology 240:849-857.

Olson L (2002) Med: clearing a path for nerve growth. Nature 416:589-590.

Pajevic S, Pierpaoli C (1999) Color schemes to represent the orientation of anisotropic tissues from diffusion tensor data: application to white matter fiber tract mapping in the human brain. Magn Reson Med 42:526-540.

Qiu Y, Wada Y, Otomo E, Tsukagoshi H (1991) Morphometric study of cervical anterior horn cells and pyramidal tracts in medulla oblongata and the spinal cord in patients with cerebrovascular diseases. J Neurol Sci 102:137-143.

Ralston DD, Ralston III HJ (1985) The terminations of corticospinal tract axons in the macaque monkey. J Comp Neurol 242:325-337.

Stejskal EO, Tanner JE (1965) Spin diffusion measurements: spin echoes in the presence of a time dependent field gradient. J Chem Phys 42:288-292.

Terashima T, Ochiishi T, Yamauchi T (1994) Immunohistochemical detection of calcium/calmodulin-dependent protein kinase II in the spinal cord of the rat and monkey with special reference to the corticospinal tract. J Comp Neurol 340:469-479.

Tsuchiya K, Fujikawa A, Suzuki Y (2005) Diffusion tractography of the cervical spinal cord by using parallel imaging. AJNR Am J Neuroradiol 26:398-400.

Tuszynski MH, Grill R, Jones LL, McKay HM, Blesch A (2002) Spontaneous and augmented growth of axons in the primate spinal cord: effects of local injury and nerve growth factor-secreting cell grafts. J Comp Neurol 449:88-101.

Yamashita Y, Takahashi M, Matsuno Y, Sakamoto Y, Oguni T, Sakae T, Yoshizumi K, Kim EE (1990) Chronic injuries of the spinal cord: assessment with MR imaging. Radiology 175:849-854. 